Europhys. Lett., 52 (5), pp. 518-524 (2000)

\title{
Coherent backscattering effect on wave dynamics in a random medium
}

\author{
H. Schomerus, K. J. H. van Bemmel and C. W. J. Beenakker \\ Instituut-Lorentz, Universiteit Leiden \\ P.O. Box 9506, 2300 RA Leiden, The Netherlands
}

(received 27 June 2000; accepted in final form 22 September 2000)

PACS. 42.25.Dd - Wave propagation in random media.

PACS. $42.25 . \mathrm{Hz}$ - Interference.

PACS. 72.15.Rn - Localization effects (Anderson or weak localization).

\begin{abstract}
A dynamical effect of coherent backscattering is predicted theoretically and supported by computer simulations: The distribution of single-mode delay times of waves reflected by a disordered waveguide depends on whether the incident and detected modes are the same or not. The change amounts to a rescaling of the distribution by a factor close to $\sqrt{2}$. This effect appears only if the length of the waveguide exceeds the localization length; there is no effect of coherent backscattering on the delay times in the diffusive regime.
\end{abstract}

Coherent backscattering refers to the systematic constructive interference of waves reflected from a medium with randomly located scatterers. The constructive interference occurs in a narrow cone around the angle of incidence, and is a fundamental consequence of time-reversal symmetry [1]. The resulting peak in the angular dependence of the reflected intensity is a generic wave effect: It has been observed using light waves [2] and acoustic waves [3], for classical and quantum scatterers [4], in passive and active media [5].

These studies mainly addressed static properties. Dynamic aspects of wave propagation in random media are now entering the focus of attention [6-9], and the work on acoustic waves [3] has started to study the connection with the coherent backscattering effect. The key observable in the dynamic experiments [6] is the derivative $\phi^{\prime}=\mathrm{d} \phi / \mathrm{d} \omega$ of the phase $\phi$ of the wave amplitude with respect to the frequency $\omega$. The quantity $\phi^{\prime}$ has the dimension of a time and is interpreted as a delay time. Van Tiggelen et al. [7] have developed a statistical theory for the distribution of the delay time $\phi^{\prime}$ and the intensity $I$ in a waveguide geometry (where angles of incidence are discretized as modes). Although the theory was worked out mainly for the case of transmission, the implications for reflection are that the distribution $P\left(\phi^{\prime}\right)$ does not depend on whether the detected mode $n$ is the same as the incident mode $m$ or not. This is in contrast with $P(I)$, which is rescaled by a factor of $1 / 2$ when $n$ becomes equal to $m$ - so that the mean $\bar{I}$ becomes twice as large. Hence it appears that no coherent backscattering effect exists for $P\left(\phi^{\prime}\right)$.

What we will demonstrate here is that this is true only if wave localization may be disregarded. Previous studies $[6,7]$ dealt with the diffusive regime of waveguide lengths $L$ below the localization length $\xi$. Here we consider the localized regime $L>\xi$ (assuming that also the 
absorption length $\xi_{\mathrm{a}}>\xi$ ). The distribution of reflected intensity is insensitive to the presence or absence of localization, being given in both regimes by Rayleigh's law:

$$
P(I)=\left\{\begin{array}{ccc}
N e^{-N I}, & \text { if } & n \neq m, \\
\frac{1}{2} N e^{-N I / 2}, & \text { if } & n=m,
\end{array}\right.
$$

(for unit incident intensity). In contrast, we find that the delay-time distribution changes markedly as one enters the localized regime, decaying more slowly for large $\left|\phi^{\prime}\right|$. Moreover, a coherent backscattering effect appears: For $L>\xi$ the peak of $P\left(\phi^{\prime}\right)$ is higher for $n=m$ than for $n \neq m$ by a factor which is close to $\sqrt{2}$. We present a complete analytical theory, compare it with numerical simulations, and offer a qualitative argument for this unexpected dynamical effect of coherent backscattering.

Let us begin with a more precise formulation of the problem. We consider a disordered medium (mean free path $l$ ) in a quasi-one-dimensional waveguide geometry (length $L$ much greater than the width $W$, with $N \gg 1$ propagating modes at frequency $\omega$ ) and study the correlator $\rho_{n m}$ of the reflected wave amplitudes at two nearby frequencies $\omega \pm \frac{1}{2} \delta \omega$,

$$
\rho_{n m}=r_{n m}\left(\omega+\frac{1}{2} \delta \omega\right) r_{n m}^{*}\left(\omega-\frac{1}{2} \delta \omega\right) .
$$

The indices $n$ and $m$ specify the detected and incident mode, respectively. (We assume singlemode excitation and detection.) The amplitudes $r_{n m}$ form the $N \times N$ reflection matrix $r$. In the localized regime (localization length $\xi \simeq N l$ smaller than both $L$ and the absorption length $\xi_{\mathrm{a}}$ ), the matrix $r$ is approximately unitary because transmission is negligibly small. We assume time-reversal symmetry (no magneto-optical effects), so that $r$ is also symmetric. Following Genack et al. $[6,7]$, we define the single-mode (or single-channel) delay time $\phi^{\prime}$ as

$$
\phi_{n m}^{\prime}=\lim _{\delta \omega \rightarrow 0} \frac{\operatorname{Im} \rho_{n m}}{\delta \omega I_{n m}},
$$

where $I_{n m}=\left|r_{n m}(\omega)\right|^{2}$ is the intensity of the reflected wave in the detected mode for unit incident intensity. In the following we will drop the indices $n$ and $m$, so as not to overburden the notation. We seek the joint distribution function $P\left(I, \phi^{\prime}\right)$ in an ensemble of different realizations of disorder.

The single-mode delay time $\phi^{\prime}$ is a linear combination of the Wigner-Smith [10] delay times $\tau_{i}(i=1,2, \ldots, N)$, which are the eigenvalues of the matrix

$$
-i r^{\dagger} \frac{\mathrm{d} r}{\mathrm{~d} \omega}=U^{\dagger} \operatorname{diag}\left(\tau_{1}, \ldots, \tau_{N}\right) U
$$

(The matrix of eigenvectors $U$ is unitary for a unitary reflection matrix.) For small $\delta \omega$ we can expand

$$
r\left(\omega \pm \frac{1}{2} \delta \omega\right)=U^{\mathrm{T}} U \pm \frac{1}{2} i \delta \omega U^{\mathrm{T}} \operatorname{diag}\left(\tau_{1}, \ldots, \tau_{N}\right) U,
$$

hence the relations

$$
\phi^{\prime}=\operatorname{Re} \frac{A_{1}}{A_{0}}, \quad I=\left|A_{0}\right|^{2}, \quad A_{k}=\sum_{i} \tau_{i}^{k} u_{i} v_{i} .
$$

We have abbreviated $u_{i}=U_{i m}, v_{i}=U_{i n}$.

The distribution of the Wigner-Smith delay times for this problem was determined recently [11]. In terms of the rates $\mu_{i}=1 / \tau_{i}$ it has the form of the Laguerre ensemble of random-matrix theory,

$$
P\left(\left\{\mu_{i}\right\}\right) \propto \prod_{i<j}\left|\mu_{i}-\mu_{j}\right| \prod_{k} \Theta\left(\mu_{k}\right) e^{-\gamma(N+1) \mu_{k}},
$$


where $\Theta(x)=1$ for $x>0$ and 0 for $x<0$. The parameter $\gamma=\alpha l / c$ (with wave velocity c) equals the scattering time, multiplied by a numerical coefficient $\alpha=\pi^{2} / 4,8 / 3$ for two-, three-dimensional scattering. (The dimensionality of the scattering inside the quasi-onedimensional waveguide is three in the experiments [6]; two-dimensional scattering applies to the computer simulations presented later, which are performed on a quasi-one-dimensional waveguide constructed from a two-dimensional lattice.) Equation (7) extends the single-mode $(N=1)$ result of refs. [12-14] to any $N$. The matrix $U$ is uniformly distributed in the unitary group. We consider first the typical case $n \neq m$ of different incident and detected modes. (The special case $n=m$ is addressed later.) For $n \neq m$ the vectors $\boldsymbol{u}$ and $\boldsymbol{v}$ become uncorrelated in the large- $N$ limit, and their elements become independent Gaussian random numbers with vanishing mean and variance $\left\langle\left|u_{i}^{2}\right|\right\rangle=\left\langle\left|v_{i}^{2}\right|\right\rangle=N^{-1}$.

It is convenient to work momentarily with the weighted delay time $W=\phi^{\prime} I$ and to recover $P\left(I, \phi^{\prime}\right)$ from $P(I, W)$ at the end. The characteristic function $\chi(p, q)=\left\langle e^{-i p I-i q W}\right\rangle$ is the Fourier transform of $P(I, W)$. The average $\langle\cdots\rangle$ is over the vectors $\boldsymbol{u}$ and $\boldsymbol{v}$ and over the set of eigenvalues $\left\{\tau_{i}\right\}$. The average over one of the vectors, say $\boldsymbol{v}$, is easily carried out, because it is a Gaussian integration. The result is a determinant,

$$
\begin{aligned}
& \chi(p, q)=\left\langle\operatorname{det}(1+i H / N)^{-1}\right\rangle, \\
& H=p \boldsymbol{u}^{*} \boldsymbol{u}^{\mathrm{T}}+\frac{1}{2} q\left(\overline{\boldsymbol{u}}^{*} \boldsymbol{u}^{\mathrm{T}}+\boldsymbol{u}^{*} \overline{\boldsymbol{u}}^{\mathrm{T}}\right) .
\end{aligned}
$$

The Hermitian matrix $H$ is a sum of dyadic products of the vectors $\boldsymbol{u}$ and $\overline{\boldsymbol{u}}$, with $\bar{u}_{i}=u_{i} \tau_{i}$, and hence has only two non-vanishing eigenvalues $\lambda_{+}$and $\lambda_{-}$. Some straightforward linear algebra gives

$$
\lambda_{ \pm}=\frac{1}{2}\left(q B_{1}+p \pm \sqrt{2 p q B_{1}+q^{2} B_{2}+p^{2}}\right)
$$

where we have defined the spectral moments

$$
B_{k}=\sum_{i}\left|u_{i}\right|^{2} \tau_{i}^{k}
$$

The resulting determinant is $\operatorname{det}(1+H / N)^{-1}=\left(1+\lambda_{+} / N\right)^{-1}\left(1+\lambda_{-} / N\right)^{-1}$, hence

$$
\chi(p, q)=\left\langle\left[1+\frac{i p}{N}+\frac{i q}{N} B_{1}+\frac{q^{2}}{4 N^{2}}\left(B_{2}-B_{1}^{2}\right)\right]^{-1}\right\rangle .
$$

An inverse Fourier transform, followed by a change of variables from $I, W$ to $I, \phi^{\prime}$, gives

$$
P\left(I, \phi^{\prime}\right)=\Theta(I)\left(N^{3} I / \pi\right)^{1 / 2} e^{-N I}\left\langle\left(B_{2}-B_{1}^{2}\right)^{-1 / 2} \exp \left[-N I \frac{\left(\phi^{\prime}-B_{1}\right)^{2}}{B_{2}-B_{1}^{2}}\right]\right\rangle .
$$

The average is over the spectral moments $B_{1}$ and $B_{2}$, which depend on the $u_{i}$ 's and $\tau_{i}$ 's via eq. (11).

This result in the localized regime is to be compared with the result of diffusion theory $[6,7]$,

$$
P_{\text {diff }}\left(I, \phi^{\prime}\right)=\Theta(I)\left(N^{3} I / \pi\right)^{1 / 2} e^{-N I}\left(Q{\overline{\phi^{\prime}}}^{2}\right)^{-1 / 2} \exp \left[-N I \frac{\left(\phi^{\prime}-\bar{\phi}^{\prime}\right)^{2}}{Q{\overline{\phi^{\prime}}}^{2}}\right] .
$$

The constants are given by $Q \simeq L / l$ and $\bar{\phi}^{\prime} \simeq L / c$ up to numerical coefficients of order unity [15]. Comparison of eqs. (13) and (14) shows that the two distributions would be 
identical if statistical fluctuations in the spectral moments $B_{1}, B_{2}$ could be ignored. However, as we shall see shortly, the distribution $P\left(B_{1}, B_{2}\right)$ is very broad, so that fluctuations cannot be ignored. The large fluctuations are a consequence of the high density of anomalously large Wigner-Smith delay times $\tau_{i}$ in the Laguerre ensemble (7), and are related to the penetration of the wave deep into the localized regions. The large $\tau_{i}$ 's are eliminated in the diffusive regime $L \lesssim \xi$, because then the finiteness of the system is felt. In that case $B_{1}$ and $B_{2}$ can be replaced by their ensemble averages, and the Gaussian theory $[6,7]$ is recovered. (The same applies if the absorption length $\xi_{\mathrm{a}} \lesssim \xi$.)

To determine how the statistical fluctuations in the spectral moments alter $P\left(I, \phi^{\prime}\right)$, we need the joint distribution $P\left(B_{1}, B_{2}\right)$. This can be calculated by applying the random-matrix technique of refs. $[16,17]$ to the Laguerre ensemble. The result is

$$
\begin{aligned}
& P\left(B_{1}, B_{2}\right)=\Theta\left(B_{1}\right) \Theta\left(B_{2}\right) \exp \left[-\frac{N B_{1}^{2}}{B_{2}}\right] \times \\
& \times\left[\frac{B_{1}^{2} \gamma N^{3}}{B_{2}^{4}}\left(B_{2}+\gamma N^{2} B_{1}\right) \exp \left[-\frac{2 \gamma N}{B_{1}}\right]-\frac{\gamma^{3} N^{5}}{4 B_{2}^{5}}\left(2 B_{2}^{2}-4 B_{1}^{2} B_{2} N+B_{1}^{4} N^{2}\right) \operatorname{Ei}\left(-\frac{2 \gamma N}{B_{1}}\right)\right],
\end{aligned}
$$

where $\operatorname{Ei}(x)$ is the exponential-integral function. The most probable values are $B_{1} \sim \gamma N$, $B_{2} \sim \gamma^{2} N^{3}$, while the mean values $\left\langle B_{1}\right\rangle,\left\langle B_{2}\right\rangle$ diverge - demonstrating the presence of large fluctuations. The distribution $P\left(I, \phi^{\prime}\right)$ follows from eq. (13) by integrating over $B_{1}$ and $B_{2}$ with weight given by eq. (15). This is an exact result in the large- $N$ limit.

For the discussion we concentrate on the distribution $P\left(\phi^{\prime}\right)=\int_{0}^{\infty} \mathrm{d} I P\left(I, \phi^{\prime}\right)$ of the singlemode delay time by itself, which takes the form

$$
P\left(\phi^{\prime}\right)=\int_{0}^{\infty} \int_{0}^{\infty} \mathrm{d} B_{1} \mathrm{~d} B_{2} \frac{P\left(B_{1}, B_{2}\right)\left(B_{2}-B_{1}^{2}\right)}{2\left(B_{2}+\phi^{\prime 2}-2 B_{1} \phi^{\prime}\right)^{3 / 2}} .
$$

We compare this distribution in the localized regime with the result of diffusion theory $[6,7]$,

$$
P_{\text {diff }}\left(\phi^{\prime}\right)=\left(Q / 2 \bar{\phi}^{\prime}\right)\left[Q+\left(\phi^{\prime} / \bar{\phi}^{\prime}-1\right)^{2}\right]^{-3 / 2}
$$

In the localized regime the value $\phi_{\text {peak }}^{\prime} \simeq \gamma N$ at the centre of the peak of $P\left(\phi^{\prime}\right)$ is much smaller than the width of the peak $\Delta \phi^{\prime} \simeq \gamma N^{3 / 2} \simeq \phi_{\text {peak }}^{\prime}(\xi / l)^{1 / 2}$. This holds also in the diffusive regime, where $\phi_{\text {peak }}^{\prime}=\bar{\phi}^{\prime}$ and $\Delta \phi^{\prime} \simeq \phi_{\text {peak }}^{\prime}(L / l)^{1 / 2}$. However, the mean $\left\langle\phi^{\prime}\right\rangle=\left\langle B_{1}\right\rangle$ diverges

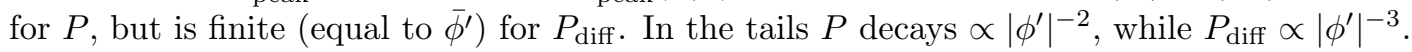

These features in the localized regime emerge in the limit $L \rightarrow \infty$ of our analytic calculations. For finite $L$ the far tail of the distribution $P\left(\phi^{\prime}\right)$ is suppressed, beyond an exponentially large cut-off at $\phi^{\prime} \gtrsim \gamma e^{L / \xi}[8]$. As a consequence, the mean delay time is finite for finite $L$ also in the localized regime, and diverges eventually in the limit $L \rightarrow \infty$.

The transition from the diffusive to the localized regime with increasing $L$ is illustrated in fig. 1. The data points are obtained from the numerical simulation of scattering of a scalar wave by a two-dimensional random medium, in a quasi-one-dimensional waveguide geometry. The reflection matrices $r\left(\omega \pm \frac{1}{2} \delta \omega\right)$ are computed by applying the method of recursive Green functions [18] to the Helmholtz equation on a square lattice (lattice constant $a$ ). The width $W=100 a$ and the frequency $\omega=1.4 c / a$ are chosen such that there are $N=50$ propagating modes. The mean free path $l=14.0 a$ is found from the formula $T=(1+s)^{-1}$ for the 

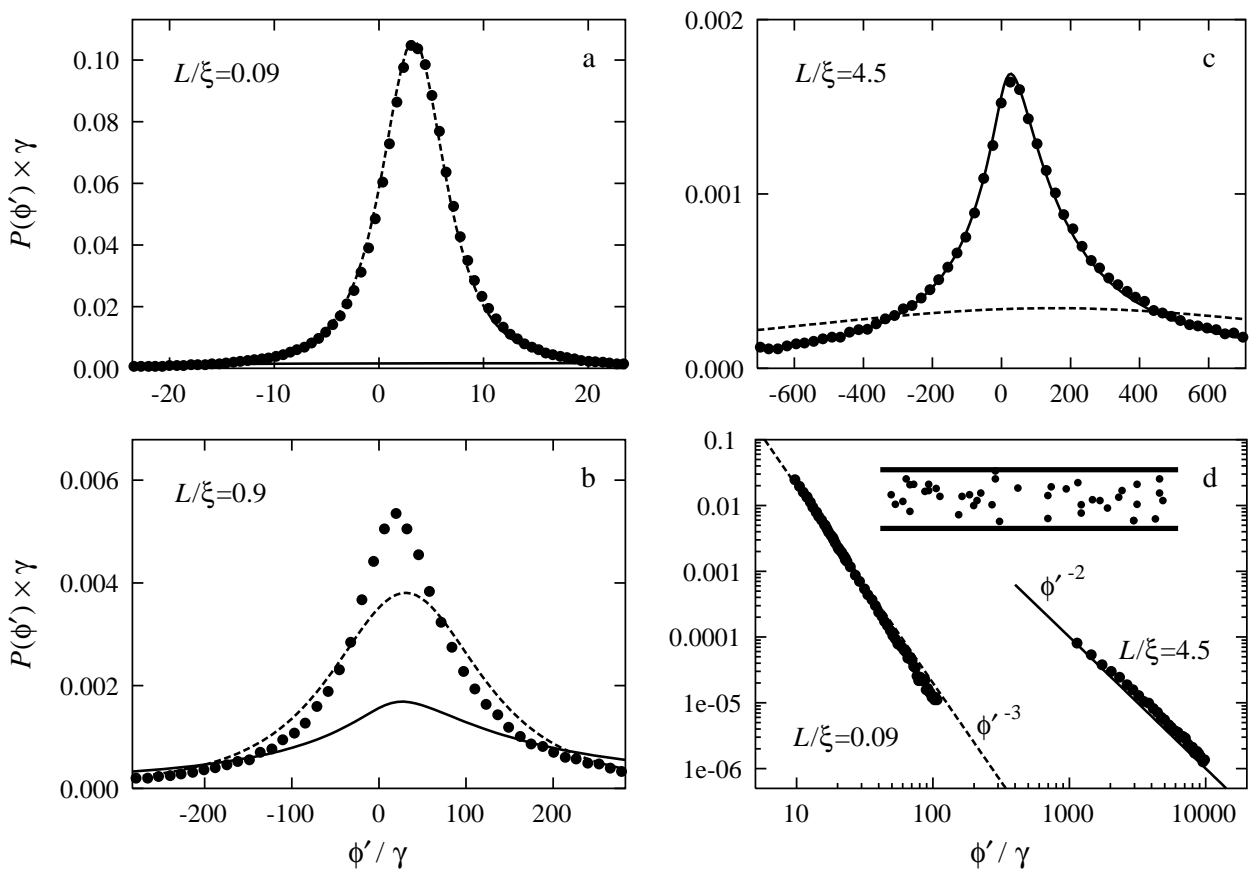

Fig. 1 - Distribution of the single-mode delay time $\phi^{\prime}$ in the diffusive regime (a), intermediate regime (b), and localized regime (c). The results of numerical simulations (data points) are compared to the prediction (17) of diffusion theory [6,7] (dashed curve) and the prediction (16) for the localized regime (solid curve). Panel (d) shows a logarithmic plot of the tails of the distributions in the diffusive and localized regime. The inset depicts a quasi-one-dimensional waveguide with randomly located scatterers. These are all results for different incident and detected modes $n \neq m$.

transmission probability in the diffusive regime $s \lesssim N$, where $s=2 L / \pi l$ for the present case of two-dimensional scattering. The corresponding localization length $\xi=N L / s=1100 a$. The parameter $\gamma=46.3 \mathrm{a} / \mathrm{c}$ is found from $\bar{\phi}^{\prime}$ in the diffusive regime [19]. The relationship between the parameters $\gamma, \bar{\phi}^{\prime}$, and $Q$ appearing in $P$ and $P_{\text {diff }}$ is given by [15]

$$
\bar{\phi}^{\prime}=\gamma \frac{s(3+2 s)}{3(1+s)}, \quad Q=\frac{8 s^{3}+28 s^{2}+30 s+15}{5(2 s+3)^{2}} .
$$

In fig. 1, the same set of parameters is used for all lengths to plot the distributions $P$ (solid curve) and $P_{\text {diff }}$ (dashed). The numerical data agrees very well with the analytical predictions in their respective regimes of validity.

We now turn to the case $n=m$ of equal-mode excitation and detection. The vectors $\boldsymbol{u}$ and $\boldsymbol{v}$ in eq. (6) are then identical, and we can write

$$
\phi^{\prime}=\operatorname{Re} \frac{C_{1}}{C_{0}}, \quad I=\left|C_{0}\right|^{2}, \quad C_{k}=\sum_{i} \tau_{i}^{k} u_{i}^{2} .
$$

The joint distribution function of the complex numbers $C_{0}$ and $C_{1}$ can be calculated in the 

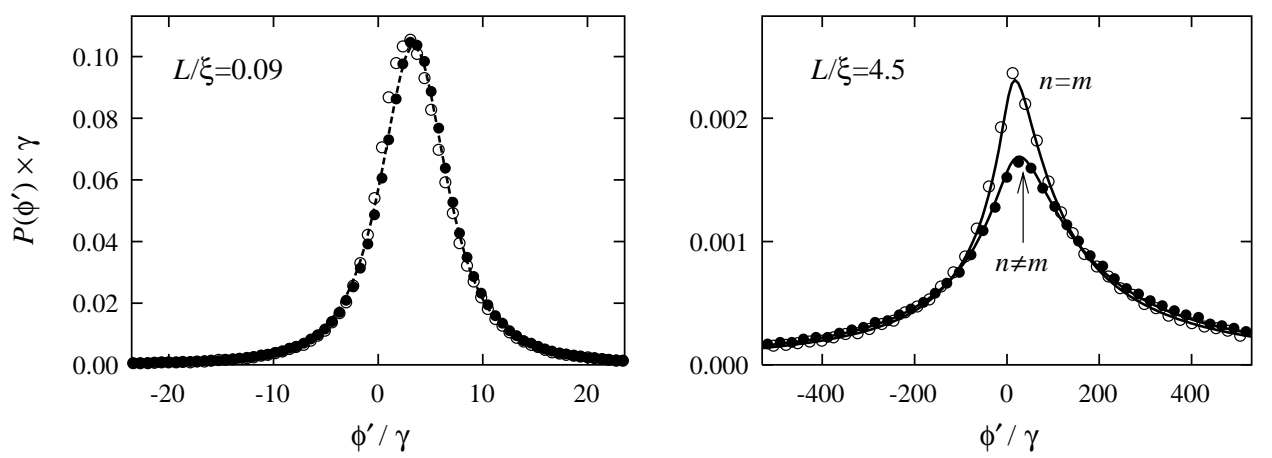

Fig. 2 - Same as fig. 1, but now comparing the case $n \neq m$ of different incident and detected modes (solid circles) with the equal-mode case $n=m$ (open circles). The curve for $n=m$ in the right panel is calculated from eqs. (19) and (20).

same way as $P\left(B_{1}, B_{2}\right)$. We find

$$
P\left(C_{0}, C_{1}\right) \propto \exp \left[-N\left|C_{0}\right|^{2} / 2\right] \int_{0}^{\infty} \mathrm{d} x x^{2} e^{-x}\left(1+\frac{\left|C_{1}\right|^{2} x^{2}}{\gamma^{2} N^{2}}-\frac{2 x}{\gamma N} \operatorname{Re} C_{0} C_{1}^{*}\right)^{-5 / 2} .
$$

The maximal value $P\left(\phi_{\text {peak }}^{\prime}\right)=\sqrt{2 / \pi N^{3} \gamma^{2}}$ for $n=m$ is larger than the maximum of $P\left(\phi^{\prime}\right)$ for $n \neq m$ by a factor $\sqrt{2} \times \frac{4096}{1371 \pi}=1.35$ in the large- $N$ limit. This is in contrast to the diffusive regime, where there is no difference in the distributions of single-mode delay times for $n=m$ and $n \neq m$. Our analytical expectations are again in excellent agreement with the numerical simulations, presented in fig. 2.

In order to explain the coherent backscattering enhancement of the peak of $P\left(\phi^{\prime}\right)$ in qualitative terms, we compare eq. (19) for $n=m$ with the corresponding relation (6) for $n \neq m$. The quantities $A_{0}$ and $A_{1}$, as well as the quantities $C_{0}$ and $C_{1}$, become mutually independent in the large- $N$ limit. (The cross-term $(\gamma N)^{-1} \operatorname{Re} C_{0} C_{1}^{*}$ in eq. (20) is of order $N^{-1 / 2}$ because $C_{0} \sim N^{-1 / 2}$ and $C_{1} \sim \gamma N$.) The main contribution to the enhancement of the peak height, namely the factor of $\sqrt{2}$, has the same origin as the factor-of-two enhancement of the mean intensity $\bar{I}$. More precisely, the relation $P\left(A_{0}\right)=\sqrt{2} P\left(\sqrt{2} C_{0}\right)$ leads to a rescaling of $P(I)$ for $n=m$ by a factor of $1 / 2$ (see eq. (1)) and to a rescaling of $P\left(\phi^{\prime}\right)$ by a factor of $\sqrt{2}$. The remaining factor of $\frac{4096}{1371 \pi}=0.95$ comes from the difference in the distributions $P\left(A_{1}\right)$ and $P\left(C_{1}\right)$. These distributions turn out to be very similar, hence the factor is close to unity. The asymptotic independence of $A_{0}$ and $A_{1}$ (as well as of $C_{0}$ and $C_{1}$ ) is another consequence of the strong fluctuations originating from the high density of anomalously large Wigner-Smith delay times $\tau_{i}$. In the diffusive regime the corresponding quantities are strongly correlated, and the coherent backscattering enhancement of the intensity affects both in the same way. Because only their ratio features in $\phi^{\prime}$, this effect cancels and no difference is observed in $P_{\text {diff }}\left(\phi^{\prime}\right)$ for $n=m$ and $n \neq m$.

In conclusion, we have discovered a dynamical effect of coherent backscattering that requires localization for its existence. Computer simulations confirm our prediction, which now awaits experimental observation.

We thank P. W. Brouwer for valuable advice. This work was supported by the Dutch Science Foundation NWO/FOM. 


\section{REFERENCES}

[1] Berkovits R. and Feng S., Phys. Rep., 238 (1994) 135.

[2] van Albada M. P. and LagendiJK A., Phys. Rev. Lett., 55 (1985) 2692; Wolf P.-E. and Maret G., Phys. Rev. Lett., 55 (1985) 2696.

[3] Bayer G. and Niederdränk T., Phys. Rev. Lett., 70 (1993) 3884; Tourin A., Derode A., Roux P., van Tiggelen B. A. and Fink M., Phys. Rev. Lett., 79 (1997) 3637.

[4] Labeyrie G., de Tomasi F., Bernard J.-C., Müller C. A., Miniatura C. and Kaiser R., Phys. Rev. Lett., 83 (1999) 5266.

[5] Wiersma D. S., van Albada M. P., van Tiggelen B. A. and Lagendijk A., Phys. Rev. Lett., 74 (1995) 4193.

[6] Genack A. Z., Sebbah P., Stoytchev M. and van Tiggelen B. A., Phys. Rev. Lett., 82 (1999) 715.

[7] van Tiggelen B. A., Sebbah P., Stoytchev M. and Genack A. Z., Phys. Rev. E, 59 (1999) 7166.

[8] Bolton-Heaton C. J., Lambert C. J., Fal'ko V. I., Prigodin V. and Epstein A. J., Phys. Rev. B, 60 (1999) 10569.

[9] Beenakker C. W. J., van Bemmel K. J. H. and Brouwer P. W., Phys. Rev. E, 60 (1999) R6313.

[10] Fyodorov Y. V. and Sommers H.-J., J. Math. Phys., 38 (1997) 1918.

[11] Beenakker C. W. J. and Brouwer P. W., to be published in Physica E.

[12] Jayannavar A. M., Vijayagovindan G. V. and Kumar N., Z. Phys. B, 75 (1989) 77.

[13] Heinrichs J., J. Phys. Condens. Matter, 2 (1990) 1559.

[14] Comtet A. and Texier C., J. Phys. A, 30 (1997) 8017.

[15] The numerical coefficients are given by $Q l / L=4 / 5 \pi, 3 / 10$ and $\bar{\phi}^{\prime} c / L=\pi / 3,4 / 3$ for two-, three-dimensional scattering. (Reference [7] gives a different result for $Q$.) These expressions are valid up to corrections of order $l / L$. More accurate formulas that account for these ballistic corrections are given in eq. (18).

[16] Fyodorov Y. V. and Sommers H.-J., Z. Phys. B, 99 (1995) 123.

[17] Schomerus H., Frahm K. M., Patra M. and Beenakker C. W. J., Physica A, 278 (2000) 469.

[18] Baranger H. U., DiVincenzo D. P., Jalabert R. A. and Stone A. D., Phys. Rev. B, 44 (1991) 10637.

[19] This value of $\gamma$ is somewhat bigger than the value $\gamma=\pi^{2} l / 4 c=34.5 a / c$ for two-dimensional scattering, as a consequence of the anisotropic dispersion relation on a square lattice. 